\title{
Neutron Halo Isomers in Stable Nuclei and their Possible Application for the Production of Low Energy, Pulsed, Polarized Neutron Beams of High Intensity and High Brilliance
}

\author{
D. Habs ${ }^{1}$, M. Gross ${ }^{1}$, P.G. Thirolf ${ }^{1}$ and P. Böni ${ }^{2}$ \\ ${ }^{1}$ Fakultät für Physik, Ludwig Maximilians Universität, München, D-85748 (Germany) \\ 2 Physik-Department E21, Technische Universität München, D-85748 Garching (Germany)
}

Received: date / Revised version: date

\begin{abstract}
We propose to search for neutron halo isomers populated via $\gamma$-capture in stable nuclei with mass numbers of about $\mathrm{A}=140-180$ or $\mathrm{A}=40-60$, where the $4 s_{1 / 2}$ or $3 s_{1 / 2}$ neutron shell model state reaches zero binding energy. These halo nuclei can be produced for the first time with new $\gamma$-beams of high intensity and small band width $(\leq 0.1 \%)$ achievable via Compton backscattering off brilliant electron beams, thus offering a promising perspective to selectively populate these isomers with small separation energies of $1 \mathrm{eV}$ to a few $\mathrm{keV}$. Similar to single-neutron halo states for very light, extremely neutron-rich, radioactive nuclei [2,3,4, the low neutron separation energy and short-range nuclear force allow the neutron to tunnel far out into free space much beyond the nuclear core radius. This results in prolonged half-lives of the isomers for the $\gamma$-decay back to the ground state in the $100 \mathrm{ps}-\mu$ s range. Similar to the treatment of photodisintegration of the deuteron, the neutron release from the neutron halo isomer via a second, lowenergy, intense photon beam has a known much larger cross section with a typical energy threshold behavior. In the second step, the neutrons can be released as a low-energy, pulsed, polarized neutron beam of high intensity and high brilliance, possibly being much superior to presently existing beams from reactors or spallation neutron sources.
\end{abstract}

\section{Introduction}

Presently, thermal and cold neutron beams are produced at large-scale facilities like reactors or spallation sources via moderation of $\mathrm{MeV}$ neutrons down to the $\mathrm{meV}$ regime. Moderators and shielding result in very large sources with $\sim 10 \mathrm{~m}$ diameter and accordingly reduced flux density. We want to realize a new intense, brilliant neutron source by first populating weakly bound, neutron halo

Send offprint requests to: isomers which, after stopping, are used to release a directed, low-energy neutron beam when irradiating them with a second laser or an X-ray beam.

Let us first compare the brilliances of X-ray beams, neutron beams, and $\gamma$ beams, which are all charge neutral. $12.4 \mathrm{keV}$ X-rays and $81.8 \mathrm{meV}$ neutrons are penetrating particles with a wavelength of $1 \AA$ that is matched to typical atomic distances in solids [5. A significant advantage of neutrons compared to X-rays is their interaction with nuclei and their magnetic moment. Hence, neutrons are deeply penetrating and can be used for the investigation of structural and magnetic volume properties of materials on a microscopic scale. In particular, neutrons interact also strongly with light elements, a property that is important for investigating soft matter and biological materials. The comparison of the XFEL [6] and the European Spallation Source ESS [7] in view of the decision to install probably both large-scale facilities in Europe indicates the complementarity between X-rays and neutron beams, although the brilliance of X-FELs $\left(10^{32} /\left(\mathrm{mm}^{2} \mathrm{mrad}^{2} \mathrm{~s}(0.1 \% \mathrm{BW})\right)\right.$ is some 30 orders of magnitude higher than that of the most intense neutron beams at the ILL reactor [8, which provides neutron beams with $10^{2}-10^{3} /\left(\mathrm{mm}^{2} \mathrm{mrad}^{2} \mathrm{~s}(0.1 \% \mathrm{BW})\right)$. The brilliance of various X-ray sources and beam lines at the ILL reactor are shown in Fig. 1]

For comparison, in Fig. 2 we have converted the neutron values of the most brilliant beam line H12 of the ILL reactor (Fig. 1b) to the standard representation used for $\mathrm{X}$ - and $\gamma$-rays, changing the neutron wavelength given in $\AA$ by the nonlinear dispersion to neutron energies given in meV by

$$
\left(\lambda_{n}[\AA]\right)^{2}=\frac{81.81}{E_{n}[\mathrm{meV}]} .
$$

The brilliances shown in Fig. 2 for the new neutron source envisaged for the ELI-Nuclear Physics project 24] using halo isomers and $\gamma$-beams will be explained later. The comparison of the different neutron brilliances points to a major breakthrough in neutron physics if 

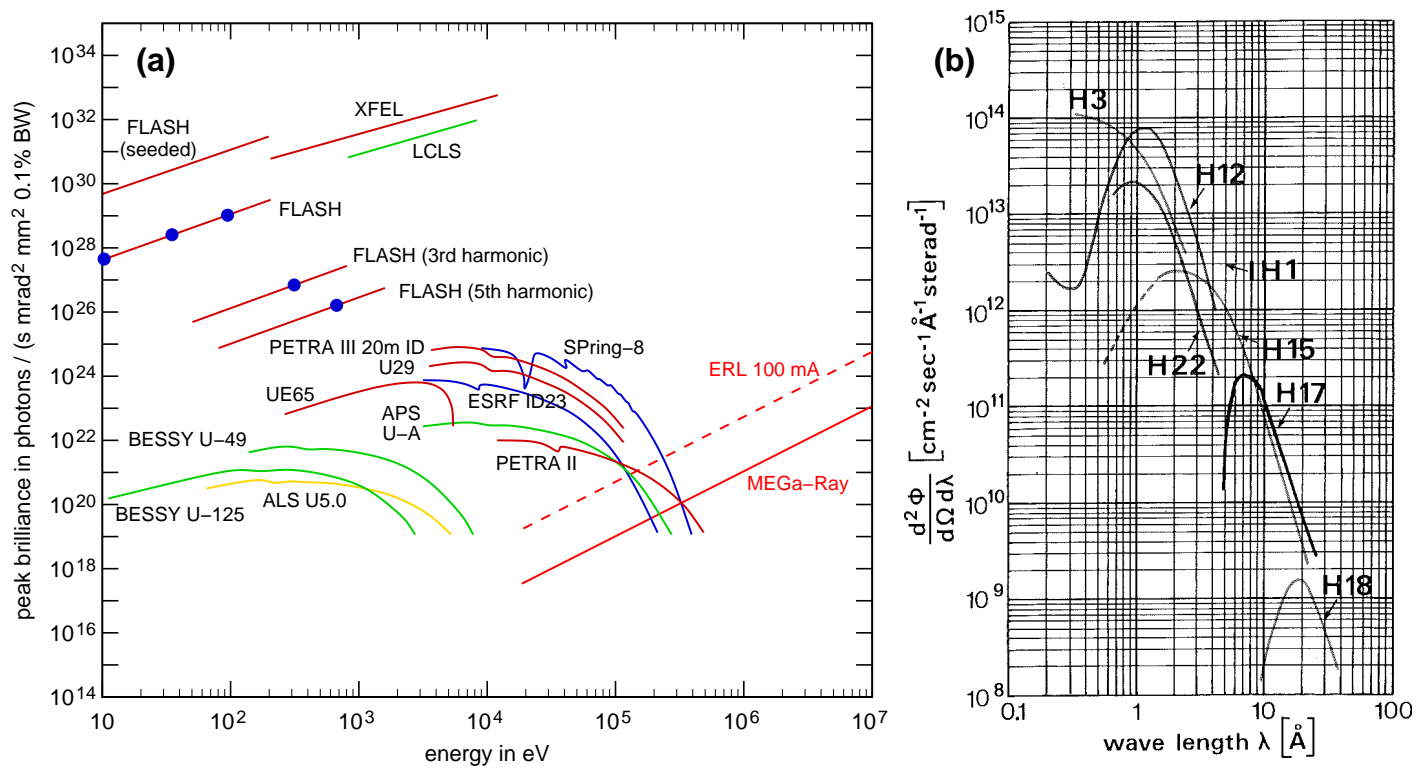

Fig. 1 (a) Brilliance of X-ray and $\gamma$-ray sources [6] 23] compared with (b) the brilliance of neutron beams at the ILL reactor (Grenoble) comprised in the Yellow Book [8] (BW=Band Width).

the two-step production process via neutron halo states proves successful.

Currently, thermal and cold neutron beams are extracted as continuous beams from nuclear reactors or as pulsed beams from spallation sources. In both cases the primarily produced neutrons have an energy of several $\mathrm{MeV}$. Therefore, to make them useful for experiments in solid-state physics, they are moderated to thermal energies by elastic scattering in heavy water (thermal neutrons), liquid hydrogen or deuterium (cold neutrons), or other moderators.
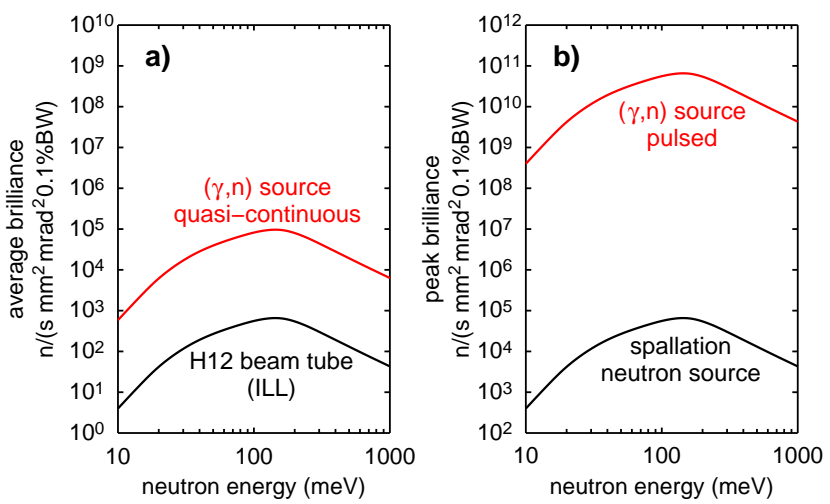

Fig. 2 (a) Average brilliance of continuous neutron sources and (b) peak brilliance of pulsed neutron sources as a function of neutron energy. For the reactor-based source we converted the curve of the H12 beam line of the ILL given in Fig. 1. For the peak brilliance of spallation sources we increased the maximum average brilliance of a reactor by a factor of 100. Only the rough energy dependence of the brilliances are relevant, since details depend on the reflectivities of neutron guides. Also the expected brilliances for the new $(\gamma, n)$ sources using halo isomers are shown in red, where the curves represent rough order of magnitude estimates.
A typical moderator of a nuclear research reactor like the FRM II in Garching or the ILL in Grenoble consists of a tank with a radius of approximately $1 \mathrm{~m}$ of heavy water, where the very small absorption cross section for neutrons and the similar masses of the neutrons and the moderator are decisive for an optimum performance of the moderation process. This tank is surrounded by a second tank of light water for shielding and cooling purposes. The neutron intensity of a high-flux reactor for neutron research is about $10^{18} \mathrm{n} / \mathrm{s}$ [5] corresponding to an optical light source or a candle of $200 \mathrm{~mW}$, if one replaces the neutrons by photons - illustrating that the presently available intensity of neutrons is very small when compared with an X-ray tube or a laser.

The neutrons are then extracted by beam tubes pointing towards the moderator followed by neutron guides. Including the biological shielding, the neutrons can be used after traversing a distance of approximately $5 \mathrm{~m}$, i.e. they exit through a surface of $\pi \cdot 10^{6} \mathrm{~cm}^{2}$, resulting in a typical overall flux $F_{\text {reac }} \simeq 3 \cdot 10^{11} \mathrm{n} /\left(\mathrm{cm}^{2} \mathrm{~s}\right)$. The neutron moderators typically emit radiation into a solid angle of $\approx 1$ sterad. If one takes this solid angle into account, this compares well with the flux of beam line $\mathrm{H} 12$ at the ILL given in the Yellow Book 8 by $F_{H 12}=2.9 \cdot 10^{10} \mathrm{n} /\left(\mathrm{cm}^{2} \mathrm{~s}\right)$.

At spallation sources, the liquid mercury target for neutron production is hit by a pulsed proton beam with a repetition rate of typically $50 \mathrm{~Hz}$ and $1 \mathrm{GeV}$ energy. It is expected that future spallation sources will reach up to $5 \mathrm{MW}$ target power, which is about an order of magnitude smaller than the thermal power of larger research reactors. The average target design flux of the ESS is $3 \cdot 10^{14} \mathrm{n} /\left(\mathrm{cm}^{2} \mathrm{~s}\right)$, which is a factor of 5 smaller than, e.g., the flux of the beam line V4 at the ILL reactor with $1.5 \cdot 10^{15} \mathrm{n} /\left(\mathrm{cm}^{2} \mathrm{~s}\right)$. Since the geometrical ex- 


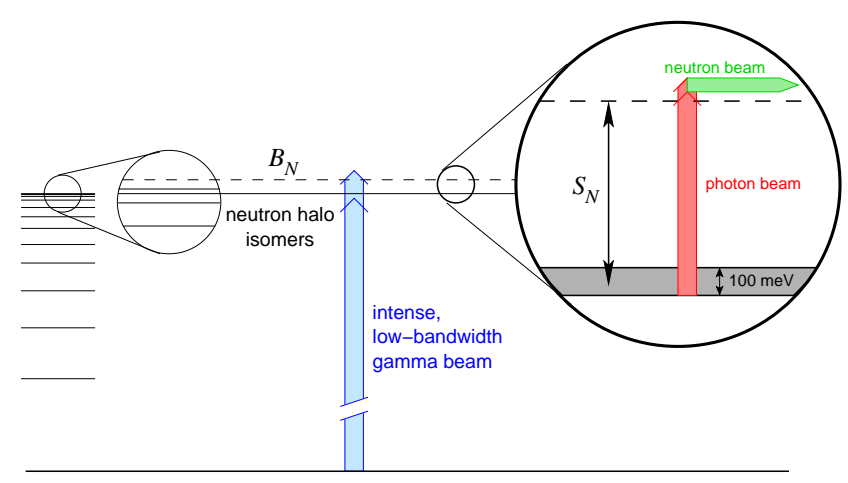

stable ground state

Fig. 3 Schematic picture of the new neutron production scheme: With the $\gamma$-beam exciting the neutron halo isomers of neutron separation energy $S_{N}$ below the binding energy $E_{B}$. The left level scheme shows the increasing number of compound nuclear reasonances with excitation energy. The halo isomer is admixed to several high-lying resonances, resulting in halo isomers with different binding energies. The two blue arrows indicate the width of the $\gamma$ beam. Then in a second step, a photon beam of much lower energy, shown in red, generates the neutron beam by dissociating the neutron halo state.

traction efficiency of a spallation source [9] is somewhat better than that of a reactor, the average extracted neutron flux may be only a factor of 2 lower than that of a reactor. However, the peak brilliance is about a factor of 100 higher 7, 15. Spallation sources as well as reactors produce large amounts of radioactivity due to the high-energy neutrons and the fission and fragmentation products. Moreover, they require large amounts of shielding, safety and security precautions. Therefore, the direct production of neutrons using $\gamma$-rays is a very attractive rather low-cost solution for the investigation of materials, when small and highly brilliant beams are required.

In Fig. 3] we show schematically the new neutron beam production scheme, where we want to produce a brilliant pulsed neutron beam directly without moderation by exciting in a first step the neutron halo isomers at excitation energies just below the neutron separation threshold via a $\gamma$-capture reaction using brilliant $\gamma$-beams of $6-8 \mathrm{MeV}$. A neutron halo isomer is a longerlived nuclear state, where one neutron of the nucleus is excited into a very weakly bound configuration, being long-lived and extending into regions far away from the nuclear core. The narrow band width of the $\gamma$-beam is essential for a selective production of the isomers. The $(\gamma, \mathrm{n})$ reaction above threshold is suppressed due to a phase space factor in neutron production. In a second step, the neutrons of these halo isomers are effectively released by a second intense laser or photon beam of narrow band width due to the large cross section, producing a brilliant neutron beam.

Such a concept only became feasible due to the development of very brilliant $\gamma$-beams, where the $\gamma$-rays

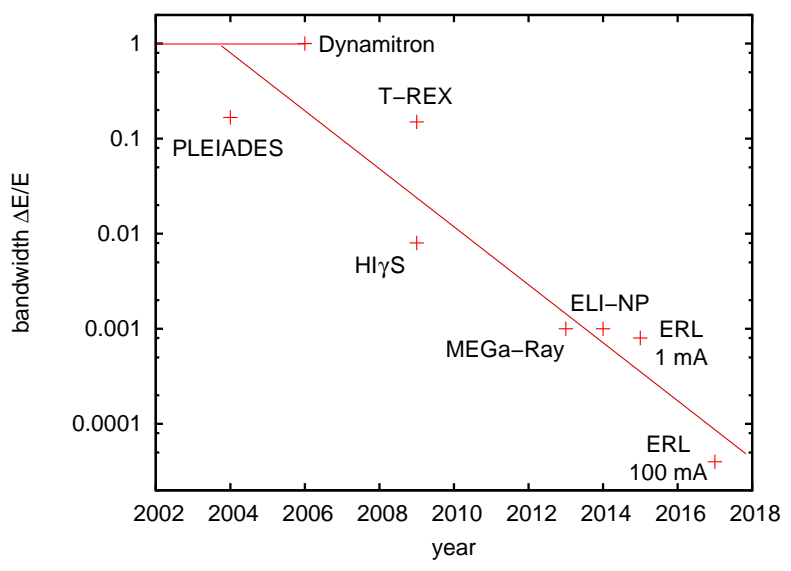

Fig. 4 Band width of high-energy $\gamma$-beams $(\approx 10 \mathrm{MeV})$ as a function of time.

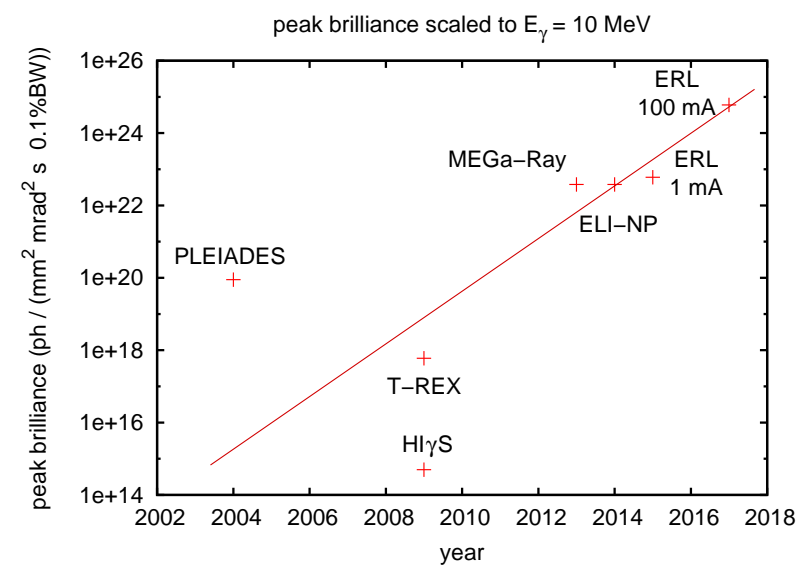

Fig. 5 Peak Brilliance of high-energy $\gamma$-beams $(\approx 10 \mathrm{MeV})$ as a function of time.

are produced by incoherent Compton back-scattering of laser light from brilliant high-energy electron bunches. Fig. 4 and Fig. 5 show the rapid progress of $\gamma$-beams with respect to band width (Fig. 4) and peak brilliance (Fig. 5) with time, starting with the bremsstrahlung spectrum of the Stuttgart Dynamitron [18, which still had a very large band width, and proceeding also to projected characteristics of future facilities (see below).

For Compton back-scattering in a head-on collision [20] the $\gamma$-energy is given by:

$$
E_{\gamma}=\frac{4 \gamma_{e}^{2} E_{L}}{1+\left(\gamma_{e} \Theta_{\gamma}\right)^{2}+4 \gamma_{e} E_{L} / m c^{2}}
$$

where the $\gamma_{e}$-factor denotes the energy of the electron beam $E_{e}=\gamma_{e} \cdot m c^{2}$, $\gamma$-energy is the energy of the $\gamma$ photon, $\Theta_{\gamma}$ is the angle between $\gamma$-beam and the the e-beam, and $E_{L}$ is the laser photon energy. The energy $E_{\gamma}$ decreases with increasing $\Theta_{\gamma}$. A small band width of the $\gamma$-beam requires a small energy spread of the electron bunches $\left(\Delta \gamma_{e} / \gamma_{e}\right)$, a small band width of the laser energy $\left(\Delta E_{L} / E_{L}\right)$, a very good emittance of the electron beam 
with a small opening and a small opening angle of the laser beam.

At the $\mathrm{HI} \gamma \mathrm{S}$ facility (Duke University, USA) the primary photons are produced by an FEL using undulators and electrons from a storage ring. Then in a second step these FEL-photons are back-scattered from the circulating electron beam 19. C. Barty and his group at the Lawrence Livermore National Laboratory (LLNL) developed already three generations of incoherent Compton back-scattering sources: PLEIADES 21, T-REX 22 and MEGa-Ray 23, each based on a "warm" electron linac and a fibre laser for back-scattering. However, the electron linac technology was switched from S-band technology $(4 \mathrm{GHz}$ ) for T-REX to X-band technology $(12 \mathrm{GHz})$ for MEGa-Ray. The MEGa-Ray $\gamma$-beam runs with a macro pulse structure of $120 \mathrm{~Hz}$ using $0.5 \mathrm{~J}, 2$ ps laser pulses, which are recirculated 100 times with 2 ns bunch spacing in a ring-down cavity. A similar $\gamma$ facility is planned for the ELI-Nuclear Physics project (ELI-NP) in Romania [24, also based on a "warm" linac like that used at MEGa-Ray, however, designed for $\gamma$ energies up to $19 \mathrm{MeV}$. At JAERI in Japan, R. Hajima and coworkers are developing a Compton back-scattering $\gamma$-beam using an energy recovery linac (ERL) and superconducting "cold" cavities [25]. For smaller electron bunch charges (10 pC), a very low normalized emittance of $0.1 \mathrm{~mm}$ mrad can be obtained from the electron gun. For the back-scattered laser light a high-finesse enhancement cavity is used for recirculating the photons. The quality of the electron beam from the ERL can be preserved by running with higher repetition rate. Switching from a $1 \mathrm{~mA}$ electron current to a $100 \mathrm{~mA}$ current, the peak brilliance and band width can be improved significantly [10,26]. Fig. 4 and Fig. 5 show the rapid improvement of the $\gamma$ beams, which will result in corresponding improvements of the neutron beams (see section 3 ).

A one-step neutron production process, i.e. releasing the neutrons in a $(\gamma, n)$ reaction just above the neutron binding energy is rather inefficient because the nucleus acquires from the high-energy $\gamma$ quantum a recoil energy $E_{r e c}$ according to

$$
E_{r e c} / A=\frac{E_{\gamma}^{2}}{2 \cdot M c^{2} \cdot A^{2}}
$$

For mass number $\mathrm{A}=180$ and $E_{\gamma}=7 \mathrm{MeV}$, we obtain $E_{\text {rec }} / A=500 \mathrm{meV}$. Thus also the emitted neutron acquires a recoil energy in $\gamma$ direction of $500 \mathrm{meV}$, which is rather large compared to the $25 \mathrm{meV}$ of thermal neutrons. Only for a very small emission opening angle, small neutron energies could be obtained, where the $\gamma$ recoil momentum is compensated. Using the neutron halo isomers, which are stopped in the target within a few ps, the $\gamma$-recoil problem can be avoided.

In the following we first (section 2) describe the expected properties of neutron halo states, their production, dissociation, and experiments for a first direct observation of neutron halo isomers in stable nuclei. In section 3 we discuss the properties of the new neutron beams like flux and brilliance. In section 4 we show possible experiments with the new neutron beam.

\section{Neutron Halo States}

The concept of a neutron halo nucleus exists for more than 50 years. A weakly bound neutron can tunnel out of the nuclear potential with the short-range forces into a large volume of free space. Due to the small binding energy the wave function by the uncertainty principle can reach out far into classically forbidden regions. A halo state is quite different from an atomic Rydberg state, where long-range Coulomb forces allow for an extended wave function of an electron with weak binding in classically allowed regions. In nuclear physics the front runner of the halo nucleus concept was the deuteron. The spin $\mathrm{S}=1$ deuteron ground state with a neutron separation energy $S_{n}=2.2246 \mathrm{MeV}$ has a halo radius a $=4.3 \mathrm{fm}$ which is much larger than the radius of $\mathrm{R}=2.0 \mathrm{fm}$ for the triplet nuclear potential between neutron and proton with a depth $V_{t}=-36 \mathrm{MeV}$ 2,11. Thus the nucleons in the deuteron spend only one third of the time within the range of the nuclear forces and two thirds of the time they are at distances larger than $\mathrm{R}$.

\subsection{Light, Extremely Neutron-rich Radioactive Neutron Halo Nuclei}

Starting in 1983 with ${ }^{11} \mathrm{Be}$ 28, and ${ }^{11} \mathrm{Li}$ 29, 30, a whole region of light, extremely neutron-rich halo nuclei was studied, establishing halos for, e.g. , ${ }^{6} \mathrm{He},{ }^{11} \mathrm{Li},{ }^{11} \mathrm{Be},{ }^{14} \mathrm{Be}$, and ${ }^{17} \mathrm{~B}[1,2,3,4$. One of the best examples for a neutron halo nucleus is ${ }^{11} \mathrm{Be}$, where the ground state $2 \mathrm{~s}_{1 / 2}^{+}$has a neutron separation energy $S_{n}=0.50 \mathrm{MeV}$ and a radius of $6.0 \mathrm{fm}$, while the first and only excited $1 p_{1 / 2}^{-}$state has a neutron separation energy $S_{n}=0.18 \mathrm{MeV}$ and a radius of $5.7 \mathrm{fm}$. These radii are large compared to the radius of the nuclear core of $\mathrm{R}=2.5 \mathrm{fm}$ 2,4 . These halo states are predominantly single particle states, where most physics is dominated by the external asymptotic wave function.

The wave function of the neutron outside the core nucleus $r \geq R$ is given by

$$
\Psi(r)=\left(\frac{\kappa}{2 \pi}\right)^{1 / 2}\left(\frac{e^{-\kappa r}}{r}\right)
$$

where the parameter $\kappa$, which has the dimension of a reciprocal length, is given by

$$
\kappa=\sqrt{2 \mu S_{n}} / \hbar=1 / a .
$$

The quantity $S_{n}$ is the neutron separation energy, $\mu$ is the reduced mass and $a$ is the scattering length. Inside a square-well potential of depth $V(r \leq R)$ the wave function of the neutron is 


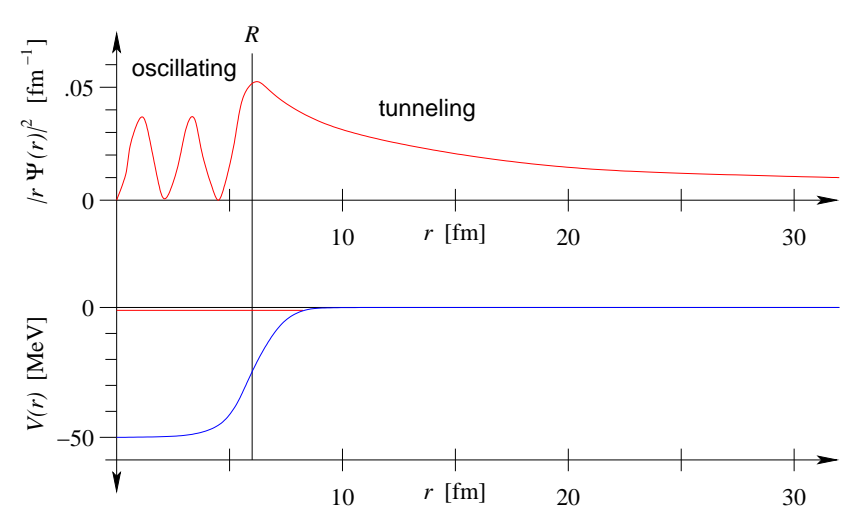

Fig. 6 Square of wave function times $r^{2}$ for Woods-Saxon potential of neutron halo state with $\mathrm{N}=4$ nodes and angular momentum $\mathrm{l}=0$. The lower part shows the potential (blue) with the weakly bound state (red).

$$
\Psi(r)=\left(\frac{\kappa}{2 \pi}\right)^{1 / 2}\left(\frac{k^{2}+\kappa^{2}}{k^{2}}\right)^{1 / 2}\left(\frac{\sin (k r)}{r}\right)
$$

with

$$
k=\sqrt{2 \mu\left(V-S_{n}\right)} / \hbar .
$$

For the large excitation energy $\left(V-S_{n}\right)$ the wave function has several nodes. For normalization of the wave function we make the approximation that the outer integral $(r \geq R)$ is much larger than the inner one. For a given potential we obtain an eigenvalue $S_{n}$. In the top part of Fig. 6 we show the squared wave function (multiplied by $r^{2}$ ) of a neutron halo state with $\mathrm{N}=4$ nodes and angular momentum $\mathrm{l}=0$, calculated for the Woods-Saxon potential indicated in the bottom part of Fig. 6.

The halo states of these extremely neutron-rich, radioactive nuclei cannot be used for a neutron source because the neutron separation energies are still a few 100 $\mathrm{keV}$ large and the target nuclei decay by very fast $\beta$ decay.

\subsection{Neutron Halo Isomers in Stable Nuclei}

Within our concept for the production of novel neutron beams we first want to search for two new islands of halo states for stable nuclei just below the neutron binding energy $S_{n}$. In these nuclei with $\mathrm{A} \approx 140-180$ or $\mathrm{A}$ $\approx 40-60$, the $4 s_{1 / 2}$, respectively $3 s_{1 / 2}$, the neutron halo state just approaches zero binding energy with a very shallow A dependence [12. This dependence can be seen in Fig. 7. We are only interested in $l=0$ neutron orbits, because only for these the centrifugal barrier vanishes. In neutron orbit calculation a central spherical WoodsSaxon potential with

$$
V(r)=-V \cdot f(r)
$$

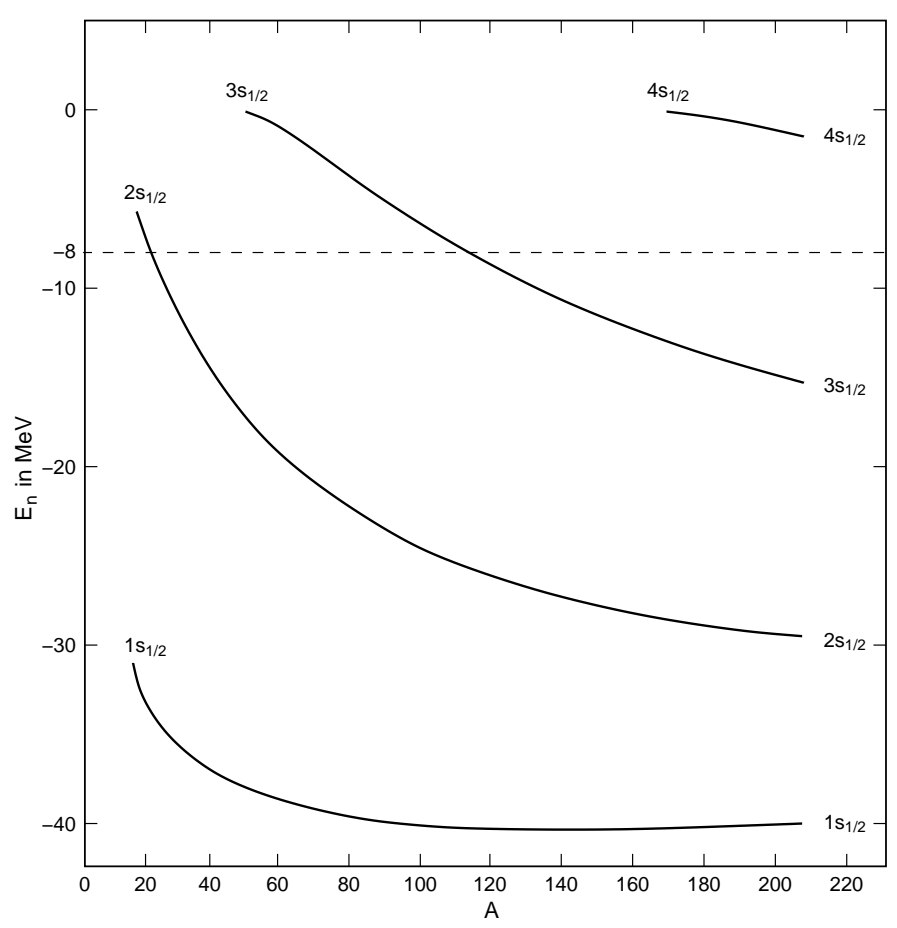

Fig. 7 Energies of neutron orbits with angular momentum $l=0$, calculated for a Woods-Saxon potential and a spin orbit $l \cdot s$ potential.

$$
\begin{gathered}
f(r)=[1+\exp ((r-R) / a)]^{-1} \\
-V=\left(-51+33 \frac{N-Z}{a}\right) M e V
\end{gathered}
$$

with $\mathrm{R}=r_{0} A^{1 / 3}$ and $r_{0}=1.25 \mathrm{fm}, \mathrm{a}=0.65 \mathrm{fm}$ is used. Also a spin orbit $\mathbf{l} \cdot \mathbf{s}$ potential was included [12]. Due to the difference in the centrifugal barrier, $l=0$ orbitals are preferred to obtain more binding for loosely bound neutrons.

In Ref. 12 also the experimental s-wave strength function measured with slow neutrons is shown as a function of mass number A. This strength function is defined as the ratio of the reduced neutron width $\Gamma_{n}^{(0)}$ divided by the average level spacing $\mathrm{D}$ and is a measure for the total amount of one-particle contribution per energy interval in the spectrum of resonances. One finds an accumulation of s-wave strength in the region $A=140-180$ and $\mathrm{A}=50-60$. Since $1 / \kappa=a$, very extended weakly bound systems have been observed in thermal neutron scattering looking at the scattering length 13 . They are compiled in Table 1, where a maximum scattering length of +49 fm corresponds to a very extended bound halo state, and was observed indirectly by neutron scattering.

Since low-energy neutron scattering and $\gamma$-capture reactions usually populate different spin and parity states, we can only predict the existence of such weakly bound neutron states in general, but nothing has been measured until now by $\gamma$-capture reactions. Thus we want to explore these states by irradiating stable nuclei with mass number $\mathrm{A}=140-180$ and $\mathrm{A}=50-60$ with $\gamma$-beams. 


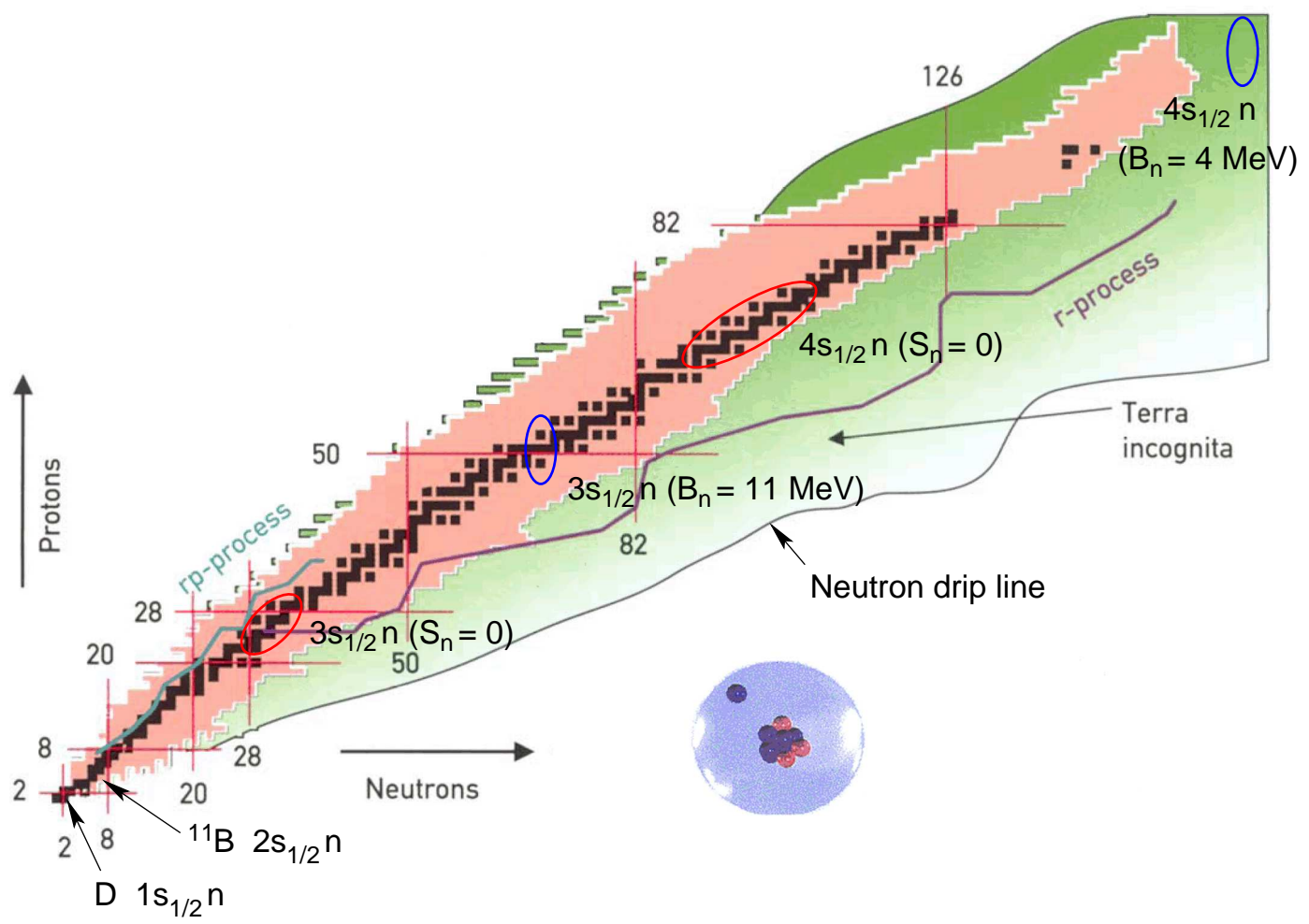

Fig. 8 Nuclidic chart with stable nuclei shown in black. The orange area contains nuclei, where some properties have been measured, while the green area called "terra incognita" covers unknown nuclei up to the neutron and proton drip line. Also indicated are the deuteron $\mathrm{D}$, the region of light, neutron-rich halo nuclei and the predicted new regions of halo nuclei (red ellipse). The excitation energies are close to the neutron binding energy, where for $A=(50-60) 3 s_{1 / 2}$ halo neutrons and for $\mathrm{A}=(150-180)$ the $4 \mathrm{~s}_{1 / 2}$ halo neutrons should lead to isomers. Also shown as blue ellipses are the regions of the $3 \mathrm{~s}_{1 / 2}$ and $4 \mathrm{~s}_{1 / 2}$ for ground state binding energies. Schematically also a 1-neutron halo nucleus with the dense core of protons and neutrons is shown at the lower right.

Table 1 Large scattering lengths $a[13$ and neutron binding energies $S_{n}$ [17] for some selected nuclei with $\mathrm{A}=140-180$ and with $\mathrm{A}=40-60$.

\begin{tabular}{ccc}
\hline isotope & $a[\mathrm{fm}]$ & $S_{n}[\mathrm{MeV}]$ \\
\hline${ }^{143} \mathrm{Nd}$ & 14.2 & 6.1 \\
${ }^{145} \mathrm{Nd}$ & 14.2 & 5.8 \\
${ }^{147} \mathrm{Pm}$ & 12.6 & 7.6 \\
${ }^{147} \mathrm{Sm}$ & 14. & 6.3 \\
${ }^{150} \mathrm{Sm}$ & 14. & 8.0 \\
${ }^{164} \mathrm{Dy}$ & 49.2 & 7.7 \\
${ }^{166} \mathrm{Er}$ & 10.5 & 8.5 \\
${ }^{174} \mathrm{Yb}$ & 19.3 & 7.5 \\
${ }^{174} \mathrm{Hf}$ & 10.9 & 8.5 \\
${ }^{180} \mathrm{Hf}$ & 13.2 & 7.4 \\
\hline${ }^{45} \mathrm{Sc}$ & 12.3 & 11.3 \\
${ }^{58} \mathrm{Fe}$ & 15. & 10.0 \\
${ }^{58} \mathrm{Ni}$ & 14.4 & 12.2 \\
\hline
\end{tabular}

Since the small binding energy $S_{n}$ of the isomers depends critically on many details like nuclear deformation, their detailed energies can only be explored experimentally. On the other hand, only the new $\gamma$-beams with very small band width allow to populate these states with sufficient selectivity to allow for their detection.

We also show these new regions of halo isomers in the nuclidic chart of Fig. 8 as red ellipses.

While for the ground state configuration one expects the $3 s_{1 / 2}$ neutron orbit close to the magic neutron number $\mathrm{N}=82$ and $\mathrm{A} \approx 138$ and the $4 s_{1 / 2}$ neutron orbit 20 neutrons below the magic neutron number $\mathrm{N}=184$ and a corresponding $\mathrm{A} \approx 270$ (blue ellipses of Fig. 8), these $3 s_{1 / 2}$ and $4 s_{1 / 2}$ neutron regions for the halo isomers with close to zero binding energies occur in very different regions. The $\mathrm{A} \approx 50$ ground state nuclei have a neutron binding energy of about $11 \mathrm{MeV}$, the $\mathrm{A} \approx 160$ nuclei of about $8.6 \mathrm{MeV}$. One can now understand the shift of the neutron orbital energy with mass number A of Fig. 7 due the the A-dependence of the Woods-Saxon potential. The radius $\mathrm{R}$ increases with $\mathrm{A}$ and also the potential $\mathrm{V}$ becomes more negative with $\mathrm{A}$, explaining this shift when solving for the eigenvalues of the $3 s_{1 / 2}$ and $4 s_{1 / 2}$ states. Thus while usually the $4 s_{1 / 2}$ ground state occurs for inaccessible super-heavy nuclei, we can study their orbits for $\mathrm{A}=140-160$ nuclei at binding energies close to zero. We can test the method for the $3 s_{1 / 2}$ orbit comparing the $\mathrm{A} \approx 50$ and $\mathrm{A} \approx 138$ regions. For odd neutron 
states close to the magic number $\mathrm{N}=82$ we can nicely identify the $3 s_{1 / 2}$ orbital.

The $4 s_{1 / 2}$ or $3 s_{1 / 2}$ states must combine with the proper spin and parity state of the core (A-1) nucleus to be excited with an E1 transition from the spin and parity of the ground state of the nucleus with mass A. Thus the neutron halo state is frequently embedded in the continuum with respect to the ground state of the (A-1) nucleus, because some excited (A-1) core states with known energy may be required to fulfill the spin and parity requirements (see Table 2 and Table 3 ).

Let us now discuss the damping between a compound nucleus resonance and a neutron halo state. We know the solution for the situation, when the $4 s_{1 / 2}$ state sits in the middle of the center of the excited compound nucleus resonance. Due to the very small energy difference in a two-state model including perturbation theory, a strong mixing occurs. We can consider the neutron as a classical particle oscillating in the deep potential of about 50 $\mathrm{MeV}$ above the bottom of the potential. However, close to the border of the Woods-Saxon potential the neutron becomes slow and needs time to explore the tunneling region. For a halo state with $1 \mathrm{eV}$ binding energy we obtain a radius $1 / \kappa \approx 4000 \mathrm{fm}$, which is extending very far out of the core by tunneling. An increase of the binding energy by $100 \mathrm{meV}$ compared to the depth of $50 \mathrm{MeV}$ and an oscillator energy $\hbar \omega_{0}=7 \mathrm{MeV}$ corresponds to a change by $\approx 10^{-8}$. Thus the wave function change inside the potential is very small and minor fluctuations in the shape of the potential e.g. of the radius can still lead to an eigenvalue at the changed energy. Thus the neutron will move a little slower inside, but will tunnel out a little less. Thus a neutron injected with a $100 \mathrm{meV}$ lower kinetic energy will have a negligible change in its oscillatory dynamics. Due to Pauli blocking, scattering in the central core is suppressed.

The damping width of the compound nucleus resonance coupling into the neutron halo isomer is large compared to the $\gamma$-decay width inside the nuclear potential (the resonance, via the strong interaction, can easily eject a quasi-free neutron), but the damping width of the halo isomer coupling back into the compound nucleus resonance is very weak, because the spatial probability for the neutron to reach an overlap with the core region again is very small. The short range nuclear interaction inside the core results in a strong coupling and fast ejection of the neutron. In the halo state the neutron is slow and with a very high probability, in free space without interaction and couples back weakly. This difference in damping and decay widths is characteristic for halo states. Usually the neutron halo state will be fragmented into many components, however, here only the few strong components are of interest, because they have several orders of magnitude higher E1 excitation strength, due to the large dipole moment of the halo state.
On the one hand, the long lifetime of the halo isomer implies a narrow width and a weak coupling to the $\approx 100 \mathrm{meV}$ [1] broad compound nucleus resonance. On the other hand, the high excitation energy of the compound nucleus resonance results in fluctuations of shape and radius of the nuclear potential, which lead to a width of the eigenvalue of the halo isomer comparable to the compound nucleus resonance [1]. The direct overlap results in strong coupling of the two states.

While the usual width is typically $\approx 100 \mathrm{meV}$, which corresponds to a lifetime of $10 \mathrm{fs}$, we expect for a halo isomer bound by $1 \mathrm{eV}$ a lifetime of about $10 \mu \mathrm{s}$ and for an isomer with $1 \mathrm{keV}$ binding energy a lifetime of about $300 \mathrm{ps}$, due to the much reduced probability in the range of the nuclear core.

Let us first make a very optimistic assumption: We find a neutron halo isomer with a binding energy $-S_{n}=$ $1 \mathrm{eV}$. Such an isomer would have a wave function extending out to $\approx 4000 \mathrm{fm}$ due to tunneling. The originally populated compound nuclear resonance, acting as a doorway state, with a typical radius of $6 \mathrm{fm}$, would expand to the neutron halo state only with a very weak overlap. While the compound states have typical $\Gamma_{\gamma}$ widths of $100 \mathrm{meV} 11$, the halo state will have a $\sim 10^{9}$ times longer half life of about $10 \mu \mathrm{s}$. The lifetimes of such neutron halo isomers are always shorter than the $\sim 900$ s lifetime of the free neutron, because the weak binding does not influence the elementary neutron decay. After populating the isomer it will lose the photon recoil momentum of (6-8) MeV/c by nuclear stopping 31 and come to rest. Since these halo isomers are still smaller than the distance between atoms in the solid, they should not be destroyed during the stopping process, where the Coulomb forces keep the nuclei apart.

So far we assumed to find a neutron halo isomer with a binding energy of $1 \mathrm{eV}$. However, we may be less lucky and only find an isomer with a larger binding energy of $1 \mathrm{keV}$. Looking at the many possibilities for the right spin combinations in the many nuclei with $A=140-180$ the chances for a $1 \mathrm{keV}$ isomer will be rather high. In this case, the extent of the neutron wave function will be a factor of 30 smaller and has a typical radius of $\sim 100$ fm. Furthermore, the isomeric lifetime will be reduced by a factor of $\sim(30)^{3}$ to about 1 ns. Everything stays the same, except that the second photon beam would not be a laser but a small second Compton backscattering X-ray beam. Even a laser-driven, relativistic electron mirror with coherent Compton back-scattering may be used [27.

The core nucleus frequently requires an excited state, to be reached from the ground state with an E1 excitation to the halo isomer, which is made by coupling the core spin and parity with the $1 / 2^{+}$halo state to the $E 1$ excitation. In a second step, the core excited state will deexcite to the ground state, leading to the final halo isomer, where the $4 s_{1 / 2}$ state is coupled to the core ground state. In Table 2 and Table 3 we have compiled 
Table 2 Table of halo isomers with stable ground state for A=140-160. The column "\%" lists the abundance of the particular isotope in $\%, I_{g}$ is the ground state spin, $I_{\text {core }}$ the spin of the (A-1) core with the known excitation energy $E_{\text {core }}$ of the excited core, $S_{n}$ are the tabulated neutron binding energies from Ref.[17].

\begin{tabular}{|c|c|c|c|c|c|c|c|c|c|c|}
\hline $\bar{A}$ & $\mathrm{Z}$ & $\%$ & $I_{g}$ & $I_{\text {core }}$ & $I_{\text {core }-g}$ & $\begin{array}{l}E_{\text {core }} \\
{[\mathrm{keV}]}\end{array}$ & $\begin{array}{c}S_{n} \\
{[\mathrm{keV}]}\end{array}$ & $\begin{array}{c}E_{t o t} \\
{[\mathrm{keV}]}\end{array}$ & $\overline{I_{i}}$ & $I_{i 0}$ \\
\hline 142 & $\mathrm{Ce}$ & 11.1 & $0^{+}$ & $3 / 2^{-}$ & $7 / 2^{-}$ & 662.06 & 7169.7 & 7831.76 & $1^{-}$ & $3^{-}$ \\
\hline 142 & $\mathrm{Ce}$ & 11.1 & $0^{+}$ & $1 / 2^{-}$ & $7 / 2^{-}$ & 1137.0 & 7169.7 & 8307. & $1^{-}$ & $4^{-}$ \\
\hline 143 & $\mathrm{Nd}$ & 12.2 & $7 / 2^{-}$ & $4^{+}$ & $0^{+}$ & 2101.32 & 6123.57 & 8224.89 & $5 / 2^{+}$ & $1 / 2^{+}$ \\
\hline 144 & $\mathrm{Nd}$ & 23.8 & $0^{+}$ & $3 / 2^{-}$ & $7 / 2^{-}$ & 742.02 & 7817.03 & 8559 . & $1^{-}$ & $3^{-}$ \\
\hline 144 & $\mathrm{Nd}$ & 23.8 & $0^{+}$ & $1 / 2^{-}$ & $7 / 2^{-}$ & 1305.82 & 7817.03 & 9123. & $1^{-}$ & $4^{-}$ \\
\hline 145 & $\mathrm{Nd}$ & 8.3 & $7 / 2^{-}$ & $4^{+}$ & $0^{+}$ & 1314.54 & 5755.29 & 7070. & $5 / 2^{+}$ & $1 / 2^{+}$ \\
\hline 146 & $\mathrm{Nd}$ & 17.2 & $0^{+}$ & $3 / 2^{-}$ & $7 / 2^{-}$ & 67.22 & 7565.23 & 7632.45 & $1^{-}$ & $3^{-}$ \\
\hline 148 & $\mathrm{Nd}$ & 5.7 & $0^{+}$ & $1 / 2^{-}$ & $5 / 2^{-}$ & 214.59 & 7332.8 & 7547.4 & $1^{-}$ & $3^{-}$ \\
\hline 148 & $\mathrm{Nd}$ & 5.7 & $0^{+}$ & $3 / 2^{-}$ & $5 / 2^{-}$ & 324.67 & 7332.8 & 7657.5 & $1^{-}$ & $2^{-}$ \\
\hline 149 & $\mathrm{Sm}$ & 13.6 & $7 / 2^{-}$ & $4^{+}$ & $0^{+}$ & 1180.26 & 5871.1 & 7051.4 & $5 / 2^{+}$ & $1 / 2^{+}$ \\
\hline 150 & $\mathrm{Nd}$ & 5.6 & $0^{+}$ & $1 / 2^{-}$ & $5 / 2^{-}$ & 165.09 & 7380.1 & 7545.2 & $1^{-}$ & $3^{-}$ \\
\hline 150 & $\mathrm{Sm}$ & 7.4 & $0^{+}$ & $3 / 2^{-}$ & $7 / 2^{-}$ & 350.04 & 7986.7 & 8336.7 & $1^{-}$ & $3^{-}$ \\
\hline 151 & $\mathrm{Eu}$ & 47.9 & $5 / 2^{+}$ & $2^{-}$ & $5^{-}$ & 70. & 7933. & 8003. & $3 / 2^{-}$ & $7 / 2^{-}$ \\
\hline 152 & $\mathrm{Sm}$ & 26.6 & $0^{+}$ & $3 / 2^{-}$ & $5 / 2^{-}$ & 4.82 & 8257.6 & 8262.4 & $1^{-}$ & $2^{-}$ \\
\hline 153 & $\mathrm{Eu}$ & 52.1 & $5 / 2^{-}$ & $3^{+}$ & $3^{-}$ & 113.97 & 8550.29 & 8664.26 & $3 / 2^{-}$ & $3 / 2^{+}$ \\
\hline 154 & $\mathrm{Sm}$ & 22.6 & $0^{+}$ & $3 / 2^{-}$ & $3 / 2^{+}$ & 35.84 & 7967.1 & 8002.9 & $1^{-}$ & $1^{+}$ \\
\hline 154 & $\mathrm{Gd}$ & 2.1 & $0^{+}$ & $3 / 2^{-}$ & $3 / 2^{-}$ & 0.0 & 8894.71 & 8894.71 & $16-$ & $1^{-}$ \\
\hline 155 & $\mathrm{Gd}$ & 14.8 & $3 / 2^{-}$ & $0^{+}$ & $0^{+}$ & 0.0 & 6435.22 & 6435.22 & $1 / 2^{+}$ & $1 / 2^{+}$ \\
\hline 156 & $\mathrm{Gd}$ & 20.6 & $0^{+}$ & $3 / 2^{-}$ & $3 / 2^{-}$ & 0.0 & 8536.39 & 8536.39 & $1^{-}$ & $1^{-}$ \\
\hline 156 & Dy & 0.1 & $0^{+}$ & $3 / 2^{-}$ & $3 / 2^{-}$ & 0.0 & 9441. & 9441. & $1^{-}$ & $1^{-}$ \\
\hline 157 & $\mathrm{Gd}$ & 15.7 & $3 / 2^{-}$ & $0^{+}$ & $0^{+}$ & 0.0 & 6359.89 & 6359.89 & $1 / 2+$ & $1 / 2^{+}$ \\
\hline 158 & $\mathrm{Gd}$ & 24.8 & $0^{+}$ & $3 / 2^{-}$ & $3 / 2^{-}$ & 0.0 & 7937.39 & 7937.39 & $1^{-}$ & $1^{-}$ \\
\hline 158 & Dy & 0.1 & $0^{+}$ & $3 / 2^{-}$ & $3 / 2^{-}$ & 0.0 & 9056. & 9056. & $1^{-}$ & $1^{-}$ \\
\hline 159 & $\mathrm{~Tb}$ & 100.0 & $3 / 2^{+}$ & $0^{-}$ & $3^{-}$ & 110.3 & 8133.1 & 8243.4 & $1 / 2^{-}$ & $7 / 2^{-}$ \\
\hline 159 & $\mathrm{~Tb}$ & 100.0 & $3 / 2^{+}$ & $1^{-}$ & $3^{-}$ & 115.5 & 8133.1 & 8248.6 & $1 / 2^{-}$ & $5 / 2^{-}$ \\
\hline
\end{tabular}

expected halo isomers. We give the excitation energy $\left(E_{t o t}, S_{n}\right)$ for the situation with an excited nuclear core and for the core ground state, assuming that the $4 s_{1 / 2}$ neutron has zero separation energy. For most nuclei the isomer may have a higher separation energy and correspondingly a lower excitation energy. For other nuclei the neutron may be unbound and no halo isomer exists. The neutron binding energies were taken from the compilation of Ref. [17. We have also listed the spins and parities of the halo isomers for the case with an excited nuclear core and the case after the deexcitation of the core. The lifetime of the core excited state will be comparable to the halo isomer lifetime.

\subsection{Photo Excitation of Neutron Halo States}

Next we estimate the cross sections for the photonuclear reaction and the width $\Gamma_{\gamma}$. The cross section for a compound nucleus resonance populated by photoexcitation at the resonance energy $E_{r}$ in the region of the neutron separation energy $S_{n}$ is given by the Breit-Wigner formula 11

$$
\sigma\left(E_{\gamma}\right)=\left(\lambda_{\gamma}^{2} / 4 \pi\right) \cdot g \cdot \frac{\Gamma_{\gamma} \Gamma_{2}}{\left(E_{\gamma}-E_{r}\right)^{2}+(\Gamma)^{2} / 4}
$$

where $g=\frac{2 I_{a}+1}{2 I_{b}+1}$ is a spin factor for the spin of the target and the beam and $\lambda_{\gamma}=(\hbar \cdot c) / E_{\gamma}$ represents the wavelength of the $\gamma$ rays with energy $E_{\gamma}$. The resonance is excited with the width $\Gamma_{\gamma}$ and decays by the width $\Gamma_{2}$. The resonance has the total width $\Gamma=\Gamma_{\gamma}+\Gamma_{2}$.

The $\Gamma_{\gamma}$ width has been studied systematically as a function of $A$ at the neutron separation energy [11 and we obtain $\Gamma_{\gamma} \approx 100 \mathrm{meV}$ for nuclei with $A=180$. The E1 hindrance factor of $\approx 10^{-5}$ is within a reasonable range but may fluctuate between different levels. Frequently the maximum cross section times the width is reported, which in our case for compound states is about $10 \mathrm{~b} \cdot \mathrm{eV}$. However, a halo state with a typical single particle E1 strength has a much larger E1 excitation width of typically $1 \mathrm{keV}$ and an integrated cross section of $10^{5} \mathrm{~b} \cdot \mathrm{eV}$. Thus even if the halo state fractionates into several components the strong components will be populated strongly. The requirement of the halo state to have a rather small binding energy limits the number of states into which it fractionates. The energy spacing of 
Table 3 Table of halo Isomers with stable ground state for $A=160-180$. The column "\%" lists the abundance of the particular isotope in $\%, I_{g}$ is the ground state spin, $I_{\text {core }}$ the spin of the (A-1) core with the known excitation energy $E_{\text {core }}$ of the excited core, $S_{n}$ are the tabulated neutron binding energies from Ref.[17].

\begin{tabular}{|c|c|c|c|c|c|c|c|c|c|c|}
\hline $\mathrm{A}$ & $\mathrm{Z}$ & $\%$ & $I_{g}$ & $I_{\text {core }}$ & $I_{\text {core-g }}$ & $\begin{array}{l}E_{\text {core }} \\
{[\mathrm{keV}]}\end{array}$ & $\begin{array}{c}S_{n} \\
{[\mathrm{keV}]}\end{array}$ & $\begin{array}{c}E_{t o t} \\
{[\mathrm{keV}]}\end{array}$ & $\overline{I_{i}}$ & $I_{i 0}$ \\
\hline 160 & Dy & 2.3 & $0^{+}$ & $3 / 2^{-}$ & $3 / 2^{-}$ & 0.00 & 8575.9 & 8575.9 & $1^{-}$ & $1^{-}$ \\
\hline 161 & Dy & 19.0 & $5 / 2^{+}$ & $1^{-}$ & $0^{+}$ & 1285.60 & 6454.39 & 7739.99 & $3 / 2^{-}$ & $1 / 2^{+}$ \\
\hline 161 & Dy & 19.0 & $5 / 2^{+}$ & $2^{-}$ & $0^{+}$ & 1264.73 & 6454.39 & 7719.12 & $5 / 2^{-}$ & $1 / 2^{+}$ \\
\hline 161 & Dy & 19.0 & $5 / 2^{+}$ & $3^{-}$ & $0^{+}$ & 1286.69 & 6454.39 & 7741.08 & $7 / 2^{-}$ & $1 / 2^{+}$ \\
\hline 162 & Dy & 25.5 & $0^{+}$ & $3 / 2^{-}$ & $5 / 2^{+}$ & 74.58 & 8196.99 & 8271.54 & $1^{-}$ & $2^{+}$ \\
\hline 162 & Dy & 25.5 & $0^{+}$ & $1 / 2^{-}$ & $5 / 2^{+}$ & 366.95 & 8196.99 & 8563.94 & $1^{-}$ & $2^{+}$ \\
\hline 163 & Dy & 24.9 & $5 / 2^{-}$ & $2^{+}$ & $0^{+}$ & 80.66 & 6271.01 & 6352.67 & $3 / 2^{+}$ & $1 / 2^{+}$ \\
\hline 164 & Dy & 28.1 & $0^{+}$ & $1 / 2^{-}$ & $5 / 2^{-}$ & 351.15 & 7658.11 & 8009.25 & $1^{-}$ & $1^{-}$ \\
\hline 164 & $\mathrm{Er}$ & 1.6 & $0^{+}$ & $1 / 2^{-}$ & $5 / 2^{-}$ & 345.60 & 8847.00 & 9192.60 & $1^{-}$ & $1^{-}$ \\
\hline 165 & Ho & 100.0 & $7 / 2^{-}$ & $4^{+}$ & $1^{+}$ & 166.1 & 7988.8 & 8154.9 & $5 / 2^{-}$ & $1 / 2^{-}$ \\
\hline 166 & Er & 33.4 & $0^{+}$ & $3 / 2^{-}$ & $5 / 2^{-}$ & 242.94 & 8474.6 & 8713.5 & $1^{-}$ & $2^{-}$ \\
\hline 167 & Er & 22.9 & $7 / 2^{+}$ & $4^{-}$ & $0^{+}$ & 1596.24 & 6436.45 & 8032.69 & $5 / 2^{-}$ & $3 / 2^{+}$ \\
\hline 168 & Er & 27.1 & $0^{+}$ & $1 / 2^{-}$ & $7 / 2^{+}$ & 207.80 & 7771.32 & 7979.12 & $1^{-}$ & $4^{-}$ \\
\hline 169 & $\mathrm{Tm}$ & 100.0 & $1 / 2^{+}$ & $1^{-}$ & $3^{+}$ & 3. & 8033.6 & 8036.6 & $1 / 2-$ & $5 / 2^{+}$ \\
\hline 170 & Er & 14.9 & $0^{+}$ & $1 / 2^{-}$ & $1 / 2^{-}$ & 0.0 & 7257.2 & 7257.2 & $1^{-}$ & $1^{-}$ \\
\hline 170 & $\mathrm{Yb}$ & 3.1 & $0^{+}$ & $3 / 2^{-}$ & $7 / 2^{+}$ & 659.63 & 8470. & 9130. & $1^{-}$ & $3^{=}$ \\
\hline 171 & $\mathrm{Yb}$ & 14.4 & $1 / 2^{+}$ & $1^{-}$ & $0^{+}$ & 1364.50 & 6614.5 & 7979. & $1 / 2^{-}$ & $1 / 2^{+}$ \\
\hline 172 & $\mathrm{Yb}$ & 21.9 & $0^{+}$ & $1 / 2^{-}$ & $1 / 2^{-}$ & 0.0 & 8019.46 & 8019.46 & $1^{-}$ & $1^{-}$ \\
\hline 173 & $\mathrm{Yb}$ & 16.2 & $5 / 2^{-}$ & $2^{+}$ & $0^{+}$ & 78.74 & 6367.3 & 6446.0 & $3 / 2^{+}$ & $1 / 2^{+}$ \\
\hline 174 & $\mathrm{Yb}$ & 31.6 & $0^{+}$ & $1 / 2^{-}$ & $5 / 2^{-}$ & 398.9 & 7464.63 & 7869.5 & $1^{-}$ & $3^{-}$ \\
\hline 175 & $\mathrm{Lu}$ & 97.4 & $7 / 2^{+}$ & $3^{-}$ & $1^{-}$ & 111.75 & 7666.7 & 7778.5 & $5 / 2^{-}$ & $1 / 2^{-}$ \\
\hline 176 & $\mathrm{Yb}$ & 12.6 & $0^{+}$ & $1 / 2^{-}$ & $7 / 2-$ & 370.89 & 6864.8 & 7235.7 & $1^{-}$ & $4^{-}$ \\
\hline 176 & $\mathrm{Hf}$ & 5.2 & $0^{+}$ & $1 / 2^{-}$ & $5 / 2^{-}$ & 125.93 & 8165.0 & 8290.9 & $1^{-}$ & $3^{-}$ \\
\hline 177 & Hf & 18.6 & $7 / 2^{-}$ & $4^{+}$ & $0^{+}$ & 290.18 & 6383.4 & 8185.4 & $1^{-}$ & $4^{-}$ \\
\hline 178 & Hf & 21.1 & $0^{+}$ & $1 / 2^{-}$ & $7 / 2^{-}$ & 559.4 & 7625.96 & 8185.4 & $1^{-}$ & $4^{-}$ \\
\hline 179 & $\mathrm{Hf}$ & 13.7 & $9 / 2^{+}$ & $4^{-}$ & $0^{+}$ & 1747.10 & 6098.99 & 7846.09 & $7 / 2^{-}$ & $1 / 2^{+}$ \\
\hline 180 & Hf & 35.2 & $0^{+}$ & $1 / 2^{-}$ & $9 / 2^{+}$ & 375.04 & 7387.78 & 7766.82 & $1^{-}$ & $5^{+}$ \\
\hline 180 & $\mathrm{~W}$ & 0.1 & $0^{+}$ & $1 / 2^{-}$ & $7 / 2^{-}$ & 221.93 & 8412. & 8634.0 & $1^{-}$ & $4^{-}$ \\
\hline
\end{tabular}

the compound nucleus resonances for a given spin and parity at the neutron binding energy for $\mathrm{A}=180$ is about $\mathrm{D} \approx 1 \mathrm{eV} 11$.

For about 60 different isotopes we have a chance of populating a halo isomer. Now the challenging question will be: what is the lowest binding energy and thus the best halo isomer? Does it have a separation energy $S_{N}$ of $1 \mathrm{eV}$ or $1 \mathrm{keV}$ ?

The Doppler broadening of a $\gamma$ transition at room temperature $k T=1 / 40 \mathrm{eV}$ for a nucleus with mass number $A=200$ and a $\gamma$ energy $E_{\gamma}=8 \mathrm{MeV}$ is

$$
\Delta E_{\gamma}=E_{\gamma} \sqrt{(2 k T) / m_{p} c^{2} A} \approx 4 \mathrm{eV}
$$

Thus the line is broadened with respect to the $\gamma$ beam by a factor of $\approx 40$. Cooling the target to low temperatures is advantageous, because then we obtain much larger maximum resonance cross sections and can use correspondingly thinner targets. On the other hand, the broadening of the second low-energy photon beam is negligible. Thus we can produce rather monoenergetic neutrons in the second excitation.

\subsection{The Photodissociation of Neutron Halo Isomers}

The photodissociation of the halo nucleus is described by the same formulas as for the deuteron [11.

$$
\sigma\left(E_{p h}\right)=\left(\frac{8 \pi}{3}\right)\left(\frac{\left((Z \cdot e)^{2} \hbar c\right.}{m_{n} c^{2} S_{n}}\right)\left(\frac{S_{n}\left(E_{p h}-S_{n}\right)}{E_{p h}^{2}}\right)^{3 / 2}
$$

Due to the change from an s- to a p-wave function, we find an energy dependence for the cross section of (excess energy above threshold $)^{3 / 2}$ from the phase space factor. The p-wave neutrons are emitted into a $\sin \theta^{2}$ cone. Due to the small separation energy $S_{n}$, and the large charge $\mathrm{Z}$, the cross section is strongly enlarged compared to the maximum cross section of the deuteron photodissociation of $2 \mathrm{mb}$. For $E_{p h}=2 S_{n}$ we reach this maximum cross section $\sigma_{\max }=\frac{\pi}{3}\left(\frac{\left((Z \cdot e)^{2} \hbar c\right.}{m_{n} c^{2} S_{n}}\right)$. For the large electric field of the coherent photon beam a much more polarized configuration with a more peaked neutron distribution is obtained. 


\subsection{Detection of Neutron Halo Isomers}

For a first identification of halo isomers, we want to measure the delayed $\gamma$ decay of the isomers and their lifetimes. Other isomers at lower excitation energies may be used for lifetime calibration. If in the future $\gamma$-beams with smaller band width become available, these halo isomers can be populated more selectively. While the prompt $\gamma$-decay of the polarized target (due to the excitation with the polarized $\gamma$-beam) shows a pronounced angular distribution with minima for the very short-lived compound nuclear resonances, the polarization of the halo isomers, due to their lifetime, will be partly lost, allowing a detection with less background in the minima of the prompt $\gamma$ angular distributions. Thus the $\gamma$-decay measurements should allow for a convincing identification of possible halo isomers.

Experimentally, we first want to identify the new class of neutron halo isomers by their unique delayed $\gamma$ cascade. Once we have identified some of these isomers, a more systematic search guided by a matched theory will become possible and we will be able to explore their properties.

In a later second step, the release of the weakly bound neutrons could be studied using a $\approx 0.5 \mathrm{keV}$ X-ray beam, produced in parallel from the electron bunches with an undulator. Here the neutron emission in the direction of the E-field of the undulator radiation, the neutron spectrum and angular distribution could be measured, leading to a first realization of this principle for a new neutron beam.

\section{Properties of the New Neutron Beam}

Let us now roughly estimate the flux and brilliance of this micro-neutron beam: While we start with $10^{13} \gamma$ quanta/s of about $7 \mathrm{MeV}$ and a band width of about 7 $\operatorname{keV}\left(\Delta E / E=10^{-3}\right)$, we excite compound nucleus resonances with a width of $\sim 100 \mathrm{meV}$ and thus should end up with an intensity of $10^{8}$ isomers/s in a target spot of $\sim 0.1 \mathrm{~mm}$ diameter. The $\mathrm{p}$-wave neutrons are emitted with $(100 \mathrm{mrad})^{2}$ opening angle and a band width better than $0.1 \%$. Thus we estimate a rough average brilliance of $\sim 10^{6} /\left[(\mathrm{mm} \mathrm{mrad})^{2} 0.1 \% \mathrm{BW} \mathrm{s}\right]$. We reduce this value by one order of magnitude to $\sim 10^{5} /\left[(\mathrm{mm} \mathrm{mrad})^{2} 0.1 \%\right.$ BW s], because we may not always reach $0.1 \%$ BW. This is about 2 orders of magnitude better than the best average brilliance of a reactor, e.g. the H12 beam line of the ILL reactor [8]. Due to the target isomer thickness of $\sim 1 \mathrm{~mm}$ (required to absorb all resonant photons of the compound nuclear resonance) and the slow movement of the neutron with about $2000 \mathrm{~m} / \mathrm{s}$, the monoenergetic neutron beam will be pulsed with $1 \mu$ s pulse duration. Thus the peak brilliance will be further increased by a factor of $10^{6}$ compared to the average brilliance reaching a peak brilliance of $\sim 10^{11} /\left[(\mathrm{mm} \mathrm{mrad})^{2} 0.1 \% \mathrm{BW}\right.$ s] (see Fig. 2).
For a $1 \mathrm{keV}$ halo isomer it would be more difficult to realize a second $\gamma$-beam with an energy of $1 \mathrm{keV}$, which strikes the target $1 \mathrm{~ns}$ after the first $\gamma$-beam of about 7 $\mathrm{MeV}$ for the production of the neutron halo isomers. In principle, such a second X-ray beam could be realized again with a band width of $10^{-3}$. Thus we would obtain a neutron beam facility with the same flux and brilliance, however requiring not a laser but a second X-ray beam.

If such a neutron beam facility would be realized, many parameters could be further optimized. The neutron pulse duration of $\sim 1 \mu$ s was determined by the target thickness. One could envision a stack of many much thinner neutron halo isomer layers, providing a macrobunch of neutrons with micro-bunches of much shorter duration, ns instead of $\mu$ s. However, then also the target thickness has to be very small (nm), to avoid a larger delay and spread of the neutron pulse when crossing the target.

The intense, brilliant second photon beam together with the large halo cross section can be used to transfer part of its high brilliance to the neutron beam. A suitable chirp of this laser can improve the band width of the neutron beam, making also use of the energy dependence of the photodissociation cross section. Also different effective target thicknesses with respect to the $\gamma$-beam and the second photon beam can improve the brilliance by tilting the target.

If we find neutron isomers with a very low binding energy, extremely monoenergetic or extremely cold neutron beams could be launched with monoenergetic laser pulses of longer duration.

For a coherent photon field of the second beam a much more directed neutron emission in the laser polarization direction may occur due to the collective alignment of the charged core nucleus with respect to the halo neutron and due to the large dipole moment, resulting in $\approx 10^{4}$ times higher brilliances. By the direction of the $E$-field of the second photon beam, releasing the neutrons, we can select into which neutron guide the neutrons are injected. For the flux and brilliance estimates of the neutron beam, we used a $\gamma$-intensity of $10^{13} \gamma$-quanta/s, while with the $100 \mathrm{~mA}$ ERL a 500 times higher flux and a 50 times smaller band width come into reach, increasing the neutron yield further by $\approx 10^{4}$. Thus $\approx 10^{8}$ times better neutron beams compared to the conservative estimates of Fig. 2 may be reached in 10 years from now (see Fig. 4 and Fig. 5 ).

It is easy to obtain without intensity loss fully polarized neutron beams. If we Compton back-scatter a fully polarized laser beam from the relativistic electron bunches, we obtain fully polarised $\gamma$-beams. If we excite from a $0^{+}$nuclear groundstate the fully polarised $1^{-}$halo isomers, we obtain fully polarized neutrons, because of their orbital angular momentum $l=0$. By switching the polarisation of the primary laser beam, we can switch from shot to shot the polarisation of the neutron beam. Since the spin of the neutron is coupled to its magnetic 
dipole moment the detailed manipulation of the polarisation of the neutron beam becomes available.

\section{Physics with the New Brilliant, Pulsed Micro-Neutron Beam}

In neutron scattering there is a large development towards cold and thermal micro-neutron beams for studying the structure and dynamics (excitations) of small samples under extreme conditions, for example in the area of solid-state and soft-matter physics. Here in particular the large field of reflectometry, small-angle neutron scattering (SANS) and diffraction will profit from highly brilliant and small beams. A large research field in fundamental physics as well as in applied physics can be opened up by such a new facility with a large user community with long-term experience in the scattering of cold and thermal neutrons.

The reasons why the interest in brilliant neutron beams with a diameter of the order of $30 \mu \mathrm{m}<d<1 \mathrm{~mm}$ has risen recently rather dramatically are manifold:

i) Modern materials exhibiting functional properties, showing for example a giant or even colossal magnetoresistance or magneto-electric coupling can often only be grown in small quantities [42]. For the investigation of the magnetic and lattice dynamical properties, however, neutron scattering is the most suitable technique.

ii) In the field of quantum phase transitions or in geoscience, samples are exposed to pressures as high as $30 \mathrm{MPa}$. Applying high pressures involves small samples. To distinguish the signals from the sample and the pressure cell, an excellent focusing of the neutron beam is required 43 . Of course, a high intensity is mandatory.

iii) Biological samples and multilayers are of major interest. Obviously, these samples can only be prepared in small quantities.

iv) A major limitation for the spatial resolution in neutron imaging is imposed by the coarse resolution of the detectors 39 . Using micron-sized beams with a large divergence would allow to apply a cone geometry as used in medicine [33, 40. The magnification of this setup helps to overcome the limited resolution of the detector.

Unfortunately, the presently available neutron beams have a low brilliance (Fig. 2), i.e. they have large cross section of typically $50 \mathrm{~mm} \times 150 \mathrm{~mm}$ and low divergence. When adapting the beam size to a typical sample size of the order of $10 \mathrm{~mm} \times 10 \mathrm{~mm}$ using focusing guides, the flux can be increased, however, due to Liouville's theorem, the divergence is increased as well [34,33. Therefore, experiments requiring an excellent momentum resolution like SANS and reflectometry cannot profit much from focusing.
It is exactly the high brilliance of the proposed $\gamma$ beam facility for the production of neutrons that may lead to major improvements in future neutron scattering techniques similarly as it occurred with the invention of synchrotron sources in the field of x-ray scattering.

As described above, the second photon beam releases the neutrons from the isomer target into a very small solid angle depending on the polarization direction of the second photon beam (Fig. 9). The neutrons will feed elliptic or parabolic neutron guides that transport the neutrons to the various beam lines for neutron scattering and imaging (Fig. 10). These neutron guides can tolerate a high neutron flux and do not transport higher energy neutrons. Since the E-field of the second photon beam oscillates into opposite directions, we always feed to oppositely directed neutron guides at the same time, which should be designed for neutron beams with similar timing and energy structure. Choppers may be used to further tailor the properties of the neutron beams. At the end of each neutron beam we place an efficient beam dump for thermal neutrons. A typical beam dump is shown in the insert of Fig. 10, where the neutrons are captured in boron carbide, while the low energy capture $\gamma$ rays $(0.5 \mathrm{MeV})$ are absorbed in tungsten. Thus even at high neutron intensities an operation without complex radiation safety licensing procedures seems possible.

Basically, for parabolic or elliptic guides the transport of the neutrons involves only one reflection in contrast to straight guides, thus minimizing the reflection losses. These guides extract neutrons very effectively, because i) only one reflection of neutrons is involved and ii) the neutron target is located in the focal point of the guide system. The second focal point of the elliptic guides will be either the position of a chopper, the position of the sample, the virtual source of a focusing monochromator or the source for conical imaging [33].

The direct line of sight to the target may be interrupted by means of a beam stop inside the guide. Thus all other radiation originating from the converter target, like $\gamma$ quanta or other neutrons are strongly suppressed. Moreover, the smearing of the time-structure of the ultra-sharp neutron beams is reduced, because the flight path of the neutrons does not depend on the divergence. For small-angle neutron scattering or reflectometry, a parabolic guide geometry is more appropriate to expand the brilliant micro-beam to a larger size and to decrease its divergence. Actually, by appropriate design of the guides, the phase space of the neutrons can be adapted to any beam size ranging from the size of the production target $d \simeq 100 \mu \mathrm{m}$ to millimeters. Using an advanced Kirkpatrick-Baez geometry for the guides [44, the beams may be effectively focused further down to a few $10 \mu \mathrm{m}$ or less.

For an efficient extraction of the neutrons, the guides have to start as close as possible to the converter target. Here, the radiation load is very high and may lead to the destruction of the glass that is presently used 
as substrate for the neutron guides. Recently it became possible to superpolish metal substrates of aluminum or iron to an atomic level [38, allowing to start with the guides very close to the target. To further reduce the background from the converter target, one may tailor the guides such that only a limited wavelength band of neutrons is transmitted. This is achieved by using bandpass supermirrors, i.e. coatings with a laterally and perpendicularly changing $d$-spacing of the multilayer structure 32 .

In order to compare the performance of the new source with a high-flux reactor, we assume that the converter target $(d \simeq 0.1 \mathrm{~mm})$ produces a neutron beam with a time averaged brilliance of $\sim 10^{5}$ neutrons $/\left[(\mathrm{mm} \mathrm{mrad})^{2}\right.$ $0.1 \% \mathrm{BW}$ s] being the source for an elliptic guide system feeding a neutron scattering experiment. The solid angle of the emitted neutrons is approximately $S_{C T}=10^{4}$ $\operatorname{mrad}^{2}$. We consider a beam line using neutrons with an energy of $81 \mathrm{meV}(\lambda=1 \AA)$. Assuming supermirrors with index $m=7$ [35, the angle of total reflection of the supermirror is $0.7^{0}$. An elliptic guide accepts neutrons with a divergence of at least $\simeq 2^{0}$, corresponding to a solid angle $S_{A}=1200 \mathrm{mrad}^{2} \ll S_{C T}$. If a beam with a bandwidth of $2 \mathrm{meV}$ is refocused on a spot with a diameter $d=0.1 \mathrm{~mm}$, we obtain a flux of $3 \cdot 10^{9} /\left[\left(\mathrm{mm}^{2}\right) \mathrm{s}\right]$ and a divergence of $2^{0}$. This is indeed an exceptionally high flux. For a beam size $d \simeq 10 \mathrm{~mm}$ a similar estimate yields a flux of $3 \cdot 10^{5} /\left[\left(\mathrm{mm}^{2}\right) \mathrm{s}\right]$ and a very small divergence of $0.02^{0}$. The upgraded triple axis spectrometer IN8 at the ILL provides a flux at the sample position of $F_{I N 8}=6.5 \cdot 10^{6} /\left[\left(\mathrm{mm}^{2}\right) \mathrm{s}\right]$ for $\lambda=1.53 \AA$, however, with a very large divergence 36 and poor energy resolution. Therefore, the converter target may be competitive with continuous sources even for the investigation of large samples. The yield for pulsed neutron beam operation will be even higher: According to Fig. 2, the converter target will deliver orders of magnitude higher intensities than spallation sources even for large beams.

Next we compare the performance of the converter target for small angle neutron scattering. Here, beams with a small divergence are essential. Assuming a brilliance of $\simeq 10^{2}$ neutrons $/\left[(\mathrm{mm} \mathrm{mrad})^{2} 0.1 \% \mathrm{BW} \mathrm{s}\right]$ at $\lambda=5 \AA$ (Fig. 2 $\mathrm{a}$ ), we end up with a flux of $3 \cdot 10^{5} /\left[\left(\mathrm{mm}^{2}\right) \mathrm{s}\right]$ for a beam size of $1 \mathrm{~mm}$, a bandwidth $\Delta \lambda / \lambda=10 \%$, and a divergence of $0.2^{0}$. For a sample size of $10 \mathrm{~mm}$, the flux

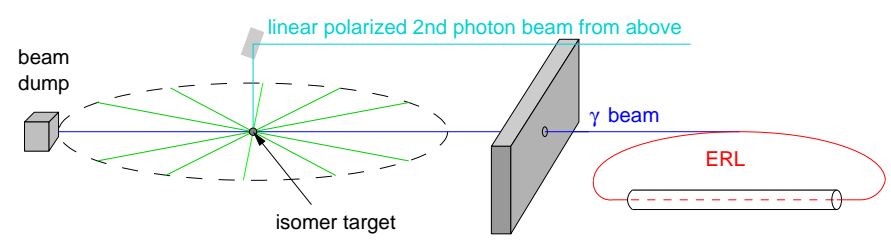

Fig. $93 \mathrm{D}$ picture showing how the second photon beam releases the neutrons from the neutron halo isomer target. The polarization of the photon beam injects the neutrons into the selected neutron guide. decreases to $3 \cdot 10^{3} /\left[\left(\mathrm{mm}^{2}\right) \mathrm{s}\right]$, however, with an excellent divergence as small as $0.02^{0}$. For comparison, the flux at the SANS-instrument KWS-1 at the FRM II is quoted to be $10^{3} /\left[\left(\mathrm{mm}^{2}\right) \mathrm{s}\right]<F<10^{5} /\left[\left(\mathrm{mm}^{2}\right) \mathrm{s}\right]$ depending on the resolution [37. Clearly, the flux of SANS at a converter target is comparable to equivalent beam lines at highflux reactors. However, we have an intrinsically pulsed beam and in 10 years from now $10^{8}$ times better values might be achieved.

The new type of neutron source can be operated in a pulsed mode as well as in a pseudo CW-mode. The former technique provides an overview of $S(\mathbf{Q}, \omega)$ in a large area of $\mathbf{Q}$ and $\omega$-space, while the latter method is usually more appropriate for the detailed investigation of $S(\mathbf{Q}, \omega)$ at particular positions in reciprocal space. Because the pulse structure and the repetition frequency of the electron and photon sources involved can be easily adjusted in a wide range of parameters, the operation of the converter target can be well adapted to the requirements of the various beam lines. By switching the polarization of the E-field of the laser during operation, the various beam lines may be fed with neutrons continuously, however, at a lower pulse rate that might reduce the problem of frame overlap. Last but not least, the possibility to fully polarize the neutrons during their production with polarized $\gamma$-rays without loss of brilliance allows to study magnetic materials and soft materials with strong incoherent scattering. By analyzing the polarization of the scattered neutrons, information on the detailed orientation of the magnetic moments in the sample and their interaction with the lattice degrees of freedom can be studied.

\section{Conclusions}

The new neutron facility opens a broad range of new possibilities for neutron physics. Once successful, neutron sources could be built even at universities resulting in a large scientific community. If one compares the investment and running costs of the proposed neutron facility with the costs of reactors or spallation sources, they will be $\approx 2$ orders of magnitude smaller. Moreover, it is also tremendously cheaper than any proposed neutron source based on inertial fusion [45]. The big advantage of the new facility is that it produces only small amounts of radioactivity and radioactive waste and thus requires only small efforts for radioprotection, i.e., very different from present reactor or spallation facilities. The new source can be operated as a multi-user neutron facility and could be several orders of magnitude more brilliant than the best existing neutron sources.

The same electron linac and recycling loop could drive in parallel a brilliant X-ray and positron source, where a $2-3 \mathrm{MeV} \gamma$-beam via the $\left(\gamma, e^{+} e^{-}\right)$reaction produces positrons in a converter target. Such a positron source would be similar to the NEPOMUC positron source 


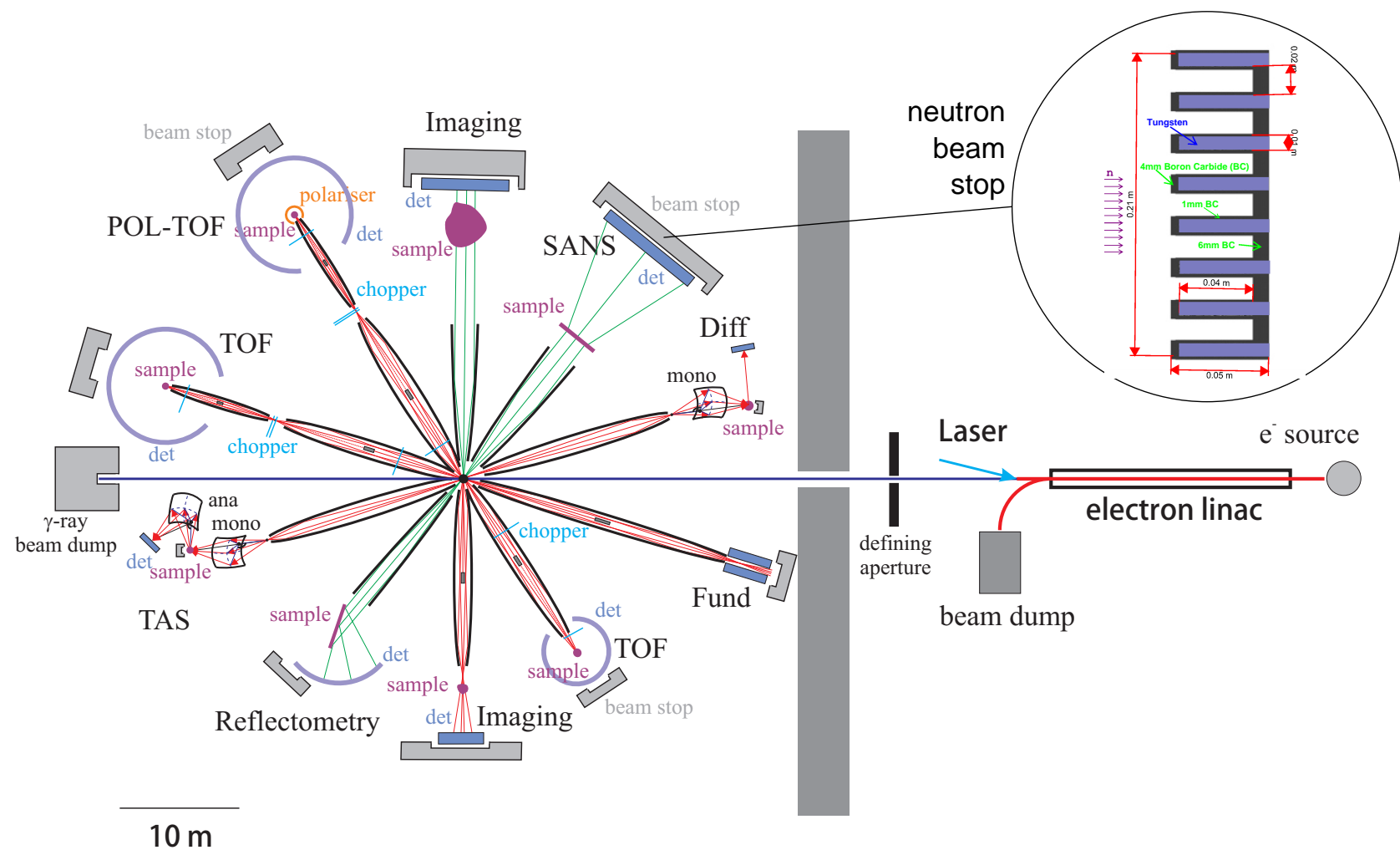

Fig. 10 Experimental setup of a brilliant pulsed micro-neutron beam facility. The intense electron beam is shown schematically in red. The brilliant $\gamma$-ray beam shown in dark blue hits the neutron converter target releasing photo neutrons. A possible arrangement of beam lines for neutron science are exemplarily shown (TOF = Time of Flight, TAS = Triple Axis Spectrometer, SANS = Small Angle Neutron Scattering camera, Diff $=$ Diffractometer, Fund $=$ Fundamental or nuclear physics $).$ Beams with low and high divergence are extracted with parabolic (green beams) or elliptic (red beams) guides, respectively.

at the FRM II [46, 47, however, the $\gamma$-rays would originate from a brilliant $\gamma$-beam and not from neutron capture. This allows to address the same targets with very different beams and analyzing techniques and makes neutron sources using halo isomers a unique possibility. In particular, these new sources have the potential, similarly as 30 years ago the synchrotron sources for X-ray scattering, to increase the flux of neutron beams by orders of magnitude in the next decades to come. Already with existing technologies, a neutron scattering facility based on halo isomers may deliver more intense beams for the investigation of small samples than today's most powerful neutron sources.

\section{Acknowledgement}

We acknowledge helpful discussions with C. Barty, R. Hajima and K. Schreckenbach. We enjoyed the close collaboration with V. Zamfir, who is heading the ELINP project. We were supported by the DFG Clusters of Excellence: Munich Centre for Advanced Photonics (MAP) and UNIVERSE.

\section{References}

1. K. Riisager, Rev. of Mod. Phys. 66, 1105-1116 (1994).

2. P.G. Hansen, A.S. Jensen and B. Jonson, Neutron Halos, Annu. Rev. Nucl. Part. Sci. 45, 591-634 (1995).

3. I. Tanihata, Neutron halo nuclei, J. Phys. G. Nucl. Part. Phys. 22, 158-198 (1996).

4. T. Aumann, A. Navin, D.P. Balamuth, D. Bazin, B. Blank, B.A. Brown, J.E. Bush, J.A. Caggiano, B. Davids, T. Glasmacher, V. Guimaraes, P.G. Hansen, R.W. Ibboton, D. Karnes, J.J. Kolata, V. Magdalena, B. Peitychenko, H. Scheit, B.M. Sherill, T.J. Totevin, One-Neutron Knockout from Individual Single-Particle States of ${ }^{11} B e$, Phys. Rev. Lett. 84, 35 (2000).

5. HERCULES Lectures, Neutron and Synchrotron Radiation for Condensed Matter Studies, Vol I, Springer Verlag, Berlin, (1994).

6. TESLA XFEL, Technical Design Report, DESY2002167, (Okt 2002).

7. F. Mezei, P. Tindemans, K. Bongardt et al., European spallation source (ESS), The 5 MW LP ESS (Feb. 2008).

8. Guide to Neutron Research facilities at the ILL, The Yellow Book, Grenoble, (1988).

9. U. Köster, private communication (2010).

10. R. Hajima, High Flux and High Brilliance $\gamma$-ray Sourcs Based on Energy Recovery Linac; http:/www.elinp.ro/excecutive committee-meeting-april12-13.php (2010). 
11. E. Segré, Nuclei and Particles, W.A. Benjamin, Inc. London (1977).

12. A. Bohr and B.R. Mottelson, Nuclear Structure, vol. I, p. 239 W.A. Benjamin, Inc., London (1969).

13. V.F. Sears, Neutron News, 3, 26-37 (1992).

14. D. Habs, T. Tajima, J. Schreiber, C.P.J. Barty, M. Fujiwara, P.G. Thirolf, Vision of nuclear physics with photonuclear reactions by laser-driven $\gamma$ Obeams, Eur. Phys. J. D 55, 279 (2009).

15. Oak Ridge spallation source (SNS), http://ornl.gov/facilities/sns-technical-parameters.shtml

16. http://www.j-parc.jp/MatLife/en/instrumentation/ns3.html

17. G. Audi, A.H. Wapstra, C. Thibault, The AME2003 atomic mass evaluation (ii). Tables, graphs and references, Nucl. Phys. A729, 337 (2003).

18. U. Kneissl, N. Pietralla, A. Zilges, J. Phys. G 32, R217 (2006).

19. H.R. Weller, M.W. Ahmed, H. Gao, W. Torrow, U.K. Wu, M. Gai, R. Miskimen, Research opportunities at the upgraded HI $\gamma$ S facility, Prog. Part. Nucl. Phys. 62, 257 (2009).

20. F.V. Hartmann, W.J. Brown, D.J. Gibson, S.G. Anderson, A.M. Tremaine, P.T. Springer, A.J. Wootton, E.P. Hartouni, C.P.J. Barty, High energy scaling of Compton scattering light sources, Phys. Rev. ST AB 8, 100702 (2005).

21. W.J. Brown, S.G. Anderson, C.P.J. Barty, S.M. Betts, R. Booth, J.K. Crane, D.N. Fiffinghoff, D.J. Gibson, F.V. Hartmann, E.P. Hartouni, J. Kuba, G.P. Le Sage, D.R. Slaughter, A.M. Tremaine, A.J. Wootton, P.T. Springer, Experimental characterization of an ultrafast Thompson scattering $X$-ray source with threedimensional time and frequency-domain analysis, Phys. Rev. ST AB 7, 060702 (2004).

22. F. Albert, S.G. Anderson, G.A. Anderson, S.M. Betts, D.J. Gibson, C.A. Hagemann, J. Hall, M.S. Johnson, M.J. Messerly, X.A. Semenov, M.Y. Shoerdin, A.M. Freimaine, F.V. Hartmann, C.W. Siders, D.P. McNabb, C.P.J. Barty, Isotope-specific detection of low-density materials with laser-based monoenergetic gamma-rays, Opt. Lett. 35, 354 (2010).

23. C. Barty, Development of MEGa-Ray technology at $L L N L$; http:/www.eli-np.ro/executive committeemeeting-april12-13.php (2010).

24. http:/www.eli-np.ro/excecutive committee-meetingapril12-13.php (2010).

25. R. Hajima, N. Kikuzawa, N. Nishimori, T. Hayakawa, T. Shizuma, K. Kawase, M. Kando, E. Minehara, H. Toyokawa, H. Ohgaki, Detection of radioactive isotopes by using Compton scattered $\gamma$-ray beams, Nucl. Instr. Meth. A608, S57 (2009).

26. V.N. Litvinenko et al., IEEE Trans. Plasma Sci. 36 17991807 (2008).

27. D. Habs, M. Hegelich, J. Schreiber, M. Gross, A. Henig, D. Kiefer, D. Jung, Dense laser-driven electron sheeets as relativistic mirrors for coherent production of brilliant $X$-ray and $\gamma$-ray beams, Appl. Phys. B 93, 349 (2008).

28. D.J. Millener, J.W. Olness, E.K. Warburton, S.S. Hanna, Strong E1 transitions in ${ }^{9} \mathrm{Be},{ }^{11} \mathrm{Be}$, and ${ }^{13} \mathrm{C}$, Phys. Rev. C 28, 497 (1983).

29. I. Tanihata, H. Hamagaki, Y. Shida, N. Yoshikawa, K. Sugimoto, O. Yamakawa, T. Kobayashi, N. Takahasi,
Measurements of Interaction Cross Sections and Nuclear Radii in the Light p-Shell Region, Phys, Rev. Lett. 55, 2676 (1985)

30. P.G. Hansen and B. Jonson, The Neutron Halo of Extremely Neutron-Rich Nuclei, Europhys. Lett. 4, 409 (1987).

31. J. F. Ziegler, J. P, Biersack, U. Littmark, The Stopping and Range of Ions in Solids, vol. 1 of series Stopping and Ranges of Ions in Matter, Pergamon Press, New York (1984).

J. F. Ziegler, Handbook of Stopping Cross Sections for Energetic Ions in All Elements, vol. 5 of series Stopping and Ranges of Ions in Matter, Pergamon Press, New York (1980).

32. M. Schneider, J. Stahn and P. Böni, Focusing of cold neutrons: Performance of a laterally graded and parabolically bent multilayer, Nucl. Instr. and Meth. A 610, 530 (2009).

33. P. Böni, New concepts for neutron instrumentation, Nucl. Instr. and Meth. A586, 1 (2008).

34. Ch. Schanzer, P. Böni, U. Filges, T. Hils, Advanced geometries for ballistic neutron guides, Nucl. Instr. and Meth. A 529, 63 (2004).

35. http://www.swissneutronics.ch/fileadmin/download/ snag_newsletter_2009-december.pdf

36. http://www.ill.eu/instruments-support/instruments-groups/instrum /in8/characteristics/

37. http://www.jcns.info/jens_kws1

38. C. Schanzer et al., Journal of Physics: Conference Series (ICNS2009), accepted for publication, (2010).

39. Conference on neutron radiograpy, Nucl. Inst. and Meth. A 542, 1 (2005).

40. N. Kardjilov, A. Hilger, M. Dawson, I. Manke, J. Banhart, M. Strobl, and P. Böni, Neutron Tomography Using an Elliptic Focusing Guide, J. Appl. Phys. 108, 034905 (2010).

41. H. Feshbach, C.E. Porter, V.F. Weisskopf, Model for Nuclear Reactions with Neutrons, Phys. Rev. 96, 448 (1954).

42. P.C. Canfield, S.L. Bud'ko, N. Ni, A. Kreyssig, A.I. Goldman, R.J. McQueeney, M.S. Torikachvilli, D.N. Argyriou, G. Luke, W. Yu, Structural, magnetic and superconducting phase transitions in $\mathrm{CaFe}_{2} \mathrm{As}_{2}$ und ambient and applied pressure, Physica C 469, 404 (2009).

43. P. G. Niklowitz, C. Pleiderer, S. Mühlbauer, P. Böni, T. Keller, P. Link, J.A. Wilson, M. Vojta, J.A. Mydosh, $\mathrm{New}$ angles on the border of antiferromagnetism in $\mathrm{NiS}_{2}$ and $\mathrm{URu}_{2} \mathrm{Si}_{2}$, Physica B 404, 2955 (2009).

44. G. E. Ice, J.W.L. Pang, C. Tulk, J. Molaison, J.-T. Choi, C. Vaughn, L. Lytle, P.Z. Takacs, K.H. Andersen, T. Bigault, A. Khounsary, Design challenges and performace of nested neutron mirrors for microfocusing on SNAP, J. Appl. Cryst. 42, 1004 (2009).

45. A. Taylor, M. Dunne, S. Bennigton, S. Ansell, I. Gardner, P. Norreys, D. Findlay,R. Nelmes, A Route to the Brightest Possible Neutron Source?, Science 315, 1092 (2007).

46. C. Hugenschmidt, G. Dollinger, W. Egger, G. Kögel, B. Löwe, J. Mayer, P. Pikart, C. Piochacz, R. Repper, K. Schrenkenbach, P. Sperr, M. Stadlbauer, Surface and bulk investigations at the high intensity positron beam facility NEPOMUC, App. Surf. Science, 255 , 29 (2008). 
47. C. Hugenschmidt, B. Löwe, J. Mayer, C. Ppiochacz, P. Pikart, R. Repper, M. Stadlbauer, K. Schreckenbach, Unprecedented intensity of a low-energy positron beam, Nucl. Instr. Meth. A 593, 616 (2008). 